$\mathbb{T}$ periodica politechnica

Civil Engineering

$51 / 1(2007)[17+24$

doi: 10.3311/pp.ci.2007-1.03

web: http://www.pp.bme.hu/ci

(c) Periodica Polytechnica 2007

RESEARCH ARTICLE

\section{Analysis of numerical differentiation methods applied for determination of kinematic velocities for LEOs}

\author{
Lóránt Földváry
}

Received 2006-11-21

\begin{abstract}
Kinematic orbits provide a time series of independent positions, which are a good base for gravity field recovery. Gravity field recovery using the energy integral requires numerical differentiation in order to get velocity information for kinetic energy. This paper deals with numerical differentiation methods to test the most effective method for velocity determination of a LEO (Low Earth Orbiter).
\end{abstract}

\section{Keywords \\ numerical differentiation · kinematic velocity}

\section{Acknowledgement}

This study was funded by the Bolyai scholarship of the Hungarian Academy of Sciences. I would like to thank for the inexpressible contribution of the researchers and professors of the Institute of Astronomical and Physical Geodesy (IAPG) at the Technical University of Munich (TUM) also.

\section{Lóránt Földváry}

MTA-BME Research Group for Physical Geodesy and Geodynamics, PO Box 91, H-1521, Hungary

e-mail: fl@ sci.fgt.bme.hu

\section{Introduction}

In the wake of the New Gravity Satellite era due to the launch of CHAMP and GRACE and in the future that of the GOCE [1], processing methods of enormously large orbit data became on the focus of geodetic interest. The input data are something else than any time before: some millions of continuous position data per satellite per year. The huge number of data arises from the continuous observation from these satellites to the GPS system. This can be done due to the much higher altitude of the GPS satellites $(20000 \mathrm{~km})$ compared to that of the gravity satellites (between 250 and $500 \mathrm{~km}$ ). The latter is often referred to as Low Earth Orbiter, i.e. LEO. The GPS-LEO constellation as described above in technical terms is called High-Low Satelliteto-Satellite Tracking (High-Low SST).

So some million position-data of LEOs are the basis of global gravity field determination techniques. The concept behind the solutions is that the satellites are in free-fall in the gravity field of the Earth. After modelling and removing all further force sources (e.g. gravitation of Sun and Moon and other planets, direct and indirect tides, surface forces (atmospheric drag, solar radiation pressure)) the remaining orbit is a trajectory in space, which is governed purely by the gravity field of the Earth. So the task is 'only' to determine the force behind the motion.

For the purpose conservation laws can be applied successfully. Newton's equation of law states the conservation of forces in a closed system. Applying it for a satellite would require information on the acceleration along the orbit. That means the use of a numerical differentiation technique twice on the orbit. Another option is the use of energy conservation law. The energy conservation law in an Earth-fixed coordinate system reads

$$
H=\frac{1}{2} \dot{x}^{2}-\frac{1}{2}(\omega \times x)^{2}-V_{p o t}
$$

with $H$ noting the Hamiltonian, $\omega$ is the angular velocity of the Earth rotation, $x$ is the position and $V_{p o t}$ is the potential. This law depends on position, $x$ (in the centrifugal term, $1 / 2(\omega \times x)^{2}$ and implicitly in the potential term, $\left.V_{p o t}\right)$ and on velocity, $\dot{x}$ (in the kinetic energy term, $1 / 2 \dot{x}^{2}$ ). Having an orbit, i.e. a time series of positions, the use of eq. (1) require derivation of velocities. In this study we focus on the velocity 
determination for the purpose of solving a gravity field model by the energy conservation law.

\section{Kinematic, Dynamic and Reduced-Dynamic Orbits}

One can distinguish three different methods of orbit determination of a LEO using high-low GPS tracking. Kinematic orbits are derived using only geometrical relationships, dynamic orbits are derived by adjusting gravity field parameters to the orbit and reduced-dynamic orbit use a given gravity model but some additional free parameters are introduced, too, in order to improve the fit of the model to the observations. Since dynamic and reduced-dynamic orbits are derived with use of a gravity field, their positions and velocities are strongly dependent on the chosen model. Therefore gravity inversion from a dynamic or reduced dynamic orbit will necessarily reflect the input gravity field.

In order to exclude such a dependency, kinematic orbits are preferable over the dynamic orbits for gravity field analysis. However kinematic orbits provide no information on velocity. These should be derived numerically from the positions.

In case of kinematic orbits, positions are derived epoch by epoch, almost independently of each other. This means that position errors are almost uncorrelated (at least, compared to the correlation between different epochs of a dynamic orbit). They exhibit an irregular pattern, when compared to a dynamic or reduced-dynamic orbit. In particular the high-frequency terms (signal and noise as well) are much smoother in the latter case. An example of typical differences of kinematic and reduceddynamic orbits is shown in Fig. 11 There are clearly jumps detectable in the figure. These jumps are, actually, some $\mathrm{cm}$ over an orbit length of $200 \mathrm{~km}$; practically these jumps are only visible in the position residuals (i.e. kinematic minus reduceddynamic positions). Since the reduced-dynamic orbit is very smooth, these jumps are mainly contributed by the kinematic orbit. Very likely these jumps are due to loss of phase connection between the GPS satellites and the receiver. These jumps occur between independently derived arcs of the orbit, reflecting the uncertainty of estimation of ambiguity parameters. In general, the continuous parts of the kinematic orbit contains useful information: purely geometrical relative positions. This can be used for velocity estimation. However, at the jumps, the edges of continuous arcs should not be employed for deriving kinematic velocities, however this is out of the scope of this paper.

\section{Methods}

First of all, we tested some numerical methods for taking the derivatives. We have tested (1) numerical differentiation, then we considered different (2) interpolation techniques followed by differentiation using (a) interpolation by fitting a higher order polynomial, (b) interpolation by cubic splines, (c) NewtonGregory interpolation, and (3) smoothing methods followed by differentiation, such as (a) smoothing by a higher order polynomial, (b) smoothing by cubic spline functions.

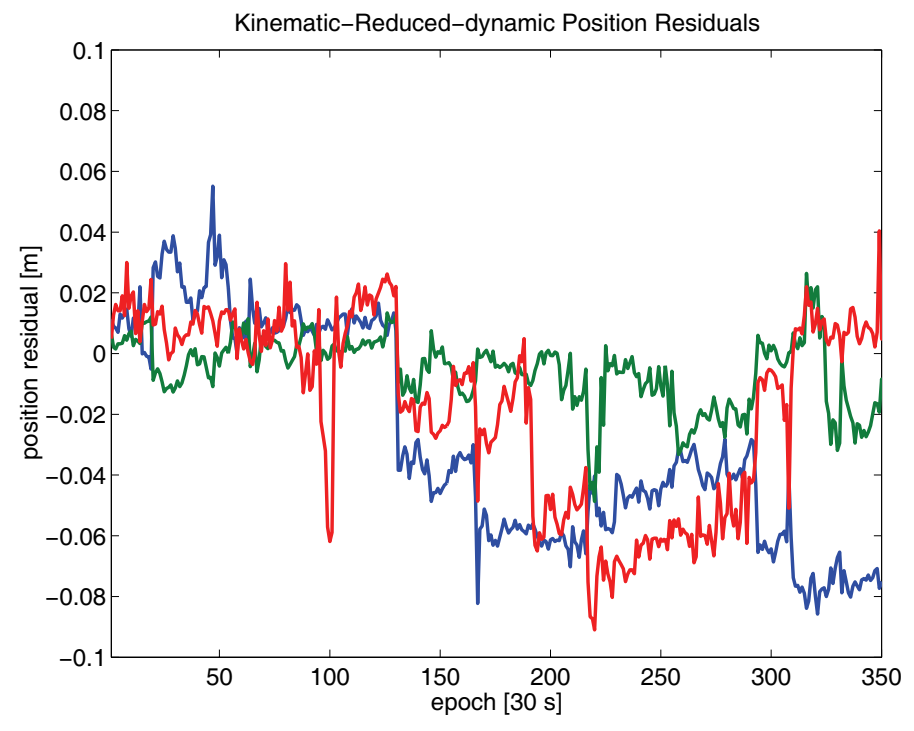

Fig. 1. Kinematic and reduced-dynamic position differences of the CHAMP satellite.

\subsection{Numerical differentiation}

Numerical differentiation in its simplest version takes the tangent of the positions at every point derived from two adjacent points:

$$
\dot{x}_{i+1 / 2}=\frac{x_{i+1}-x_{i}}{t_{i+1}-t_{i}}
$$

The tangent determined linearly from two points, the $i+1$ th and the $i$ th points, provides an estimation of the derivative at the midpoint, i.e. at the $i+1 / 2$ th epoch. The derivatives derived this way, therefore, have to be shifted to discrete points, epochs with integer indexes. This is done by linear interpolation. For uniformly distributed time series, i.e. $d t=t_{i+1}-t_{i}=$ constant, the velocity reads

$$
\dot{x}_{i}=\frac{\dot{x}_{i+1 / 2}+\dot{x}_{i-1 / 2}}{2}=\frac{x_{i+1}+x_{i-1}}{2 \cdot \mathrm{d} t} .
$$

\subsection{Interpolation by Fitting a Higher Order Polynomial}

Let us define an $n-1$ th order polynomial at an arbitrary point $i$ of the time series

$$
x_{i}=a_{n-1} t_{i}^{n-1}+a_{n-2} t_{i}^{n-2}+\cdots+a_{2} t_{i}^{2}+a_{1} t_{i}+a_{0} .
$$

An $n-1$ th order polynomial can unequivocally be fitted to $n$ consecutive points by solving the coefficients of the polynomial, $a_{i}$, with $i=0, \ldots, n-1$, because the number of unknowns and the number of equations are equal. Then the time derivative can be analytically derived as

$$
\dot{x}_{i}=(n-1) a_{n-1} t_{i}^{n-2}+(n-2) a_{n-2} t_{i}^{n-3}+\cdots+2 a_{2} t_{i}+a_{1} .
$$

In this study we have used 7 th order polynomial for deriving velocities, using 8 points for the estimation of the coefficients. The polynomial estimation is done piece-wise: in any $i$ th point the neighbouring points $i-3$ th, $i-2$ th, .., $i+3$ th, $i+4$ th are used for estimating the polynomial. At the end of arcs, i.e. in the first three and the last four points of a continuous arc, the velocity is estimated from the polynomial derived in the fourth and in the last-minus-fifth points, respectively. 


\subsection{Interpolation by Cubic Splines}

In general, a cubic spline is a set of piece-wise cubic polynomials with certain boundary conditions. Since it is an interpolation technique, at discrete points of the curve the spline function, $g(t)$, is equal to the corresponding value of the time series, $g\left(t_{i}\right)=x_{i}$. The function is 'piece-wise', i.e. an epochby-epoch piece-wise function, so every cubic function is valid only within one interval of the time series (i.e. between $t_{i+1}$ and $\left.t_{i}\right)$, i.e. $g\left(t_{i}\right)=x(t)$ for $t_{i} \leq t<t_{i+1}$.

We are interested in those piece-wise cubic functions, that fulfil the following properties: The cubic function must be (1) continuous, i.e. $g\left(t_{i}\right)_{-}=g\left(t_{i}\right)_{+}$at every $i$. It should satisfy (2) the first and (3) the second derivatives as well, i.e. $\dot{g}\left(t_{i}\right)_{-}=\dot{g}\left(t_{i}\right)_{+}$ and $\ddot{g}\left(t_{i}\right)_{-}=\ddot{g}\left(t_{i}\right)_{+}$. These properties provide continuity at the edges of the pieces, i.e. at the discrete points. (4) The third derivative should be equivalent with the step function. Therefore it is also continuous in $\left[t_{i}, t_{i+1}\right]$ for any discrete $i$, but the lower limit and the upper limit are not necessarily equal, $\ddot{g}\left(t_{i}\right)_{-} \neq \ddot{g}\left(t_{i}\right)_{+}$.

Among these sets of continuous piece-wise cubic functions the natural cubic spline is that particular one, which minimizes the second derivative, that is

$$
\int_{t_{0}}^{t_{n}} \ddot{g}(t) \mathrm{d} t=\min .
$$

It minimizes the curvature of the function $g(t)$.

Finding the one and only solution of $g(t)$ satisfying the continuity properties and the minimum condition, one ends up with cubic functions for every single interval $\left[t_{i}, t_{i+1}\right]$, for any $i$ it reads

$$
g(t)=a_{i} t^{3}+b_{i} t^{2}+c_{i} t+d_{i}
$$

for $t_{i} \leq t<t_{i+1}$. The derivative can then be derived analytically as

$$
\dot{g}(t)=3 a_{i} t^{2}+2 b_{i} t+c_{i} .
$$

The exact formulations solving for the vectors of coefficients ( $a$, $b, c$ and $d$ ) can be found in [6].

\subsection{Newton-Gregory Interpolation}

An $n-1$ th order polynomial at an arbitrary point $i$ of a time series reads (cf. subsection 3.2. (Eq.) 44 $x_{i}=a_{n-1} t_{i}^{n-1}+$ $a_{n-2} t_{i}^{n-2}+\cdots+a_{2} t_{i}^{2}+a_{1} t_{i}+a_{0}$. This equation contains $n$ unknowns, the coefficients $a_{i}$ from $i=0, \ldots, n-1$. By considering $n$ neighbouring points, one can solve for these coefficients. In an equivalent form it can be written as

$$
\begin{aligned}
x_{i}= & a_{0}+a_{1}\left(t_{i}-t_{1}\right)+a_{2}\left(t_{i}-t_{1}\right)\left(t_{i}-t_{2}\right)+\cdots \\
& +a_{n-1}\left(t_{i}-t_{1}\right)\left(t_{i}-t_{2}\right) \ldots\left(t_{i}-t_{n-1}\right),
\end{aligned}
$$

where $t_{1}, t_{2}, \ldots, t_{n-1}$ are the known values of the interpolation (i.e. the existing entries of the time series) and $t_{i}$ is the "running' point, at which the interpolation should be evaluated. It is equivalent with the polynomial in that both forms describe the orbit with powers of $t$ up order $n-1$ and $n$ coefficients $a_{i}$. The number of unknowns, $a_{i}$, is $n$, so that we can unequivocally determine them by considering $n$ neighboring points of the time series.

The solution can be obtained by solving for the increasing orders of $i$. The coefficients at the first points are

$$
a_{0}=x_{1}, a_{1}=\frac{x_{2}-x_{1}}{t_{2}-t_{1}}, a_{2}=\frac{\frac{x_{3}-x_{1}}{t_{3}-t_{1}}-\frac{x_{2}-x_{1}}{t_{2}-t_{1}}}{t_{3}-t_{2}} .
$$

The 0 th coefficient, $a_{0}$, is equal to $x$ itself and can be explained as the 0th gradient of $x$. The $a_{1}$ coefficient is the first order gradient, while $a_{2}$ is the gradient of the second order gradient. Therefore the $n$th coefficient can be generalized as the $n$th gradient of the function.

By assuming equidistant abcissae, $t$, the solution gets simpler, since any $t_{i+1}-t_{i}$ time difference is a constant, $h$, and it redefines the $n$th order gradient as an $n$th order difference of the function in the nominator and the $n$th power of the time difference, $h$, and the factorial of the order in the denominator:

$$
a_{i}=\frac{1}{n !} \frac{\Delta^{n} x_{0}}{h^{n}}
$$

After obtaining the coefficients using the equation above, the first derivative of a general $j$ th tag of the interpolation formula, $a_{j-1}\left(t_{i}-t_{1}\right)\left(t_{i}-t_{2}\right) \ldots\left(t_{i}-t_{j-1}\right)$ becomes

$$
\begin{aligned}
& a_{j-1}\left[\left(t_{i}-t_{2}\right)\left(t_{i}-t_{3}\right) \ldots\left(t_{i}-t_{j-1}\right)\right. \\
& \quad+\left(t_{i}-t_{1}\right)\left(t_{i}-t_{3}\right) \ldots\left(t_{i}-t_{j-1}\right)+\cdots \\
& \left.\quad+\left(t_{i}-t_{1}\right)\left(t_{i}-t_{2}\right) \ldots\left(t_{i}-t_{j-2}\right)\right] ;
\end{aligned}
$$

or equivalently in a compact notation

$$
a_{j-1} \sum_{k=1}^{j-1} \prod_{f=1}^{j-1}\left(t_{i}-t_{f}\right)
$$

for $f \neq k$.

In this study we used 7 adjacent points for the interpolation. In order to represent the numerical simplicity of this method, we show the solution of the first time derivative of the function $x$, $\dot{x}_{i}=\frac{1}{60 h}\left(-x_{i-3}+9 x_{i-2}-45 x_{i-1}+45 x_{i+1}-9 x_{i+2}+x_{i+3}\right)$.

\subsection{Smoothing by a Higher Order Polynomial}

In general, the 'smoothing' method is the same what we call 'interpolation' using a higher order polynomial (cf. section 3.2), with a solution for the derivative $\dot{x}_{i}=(n-1) a_{n-1} t_{i}^{n-2}[1]+$ $(n-2) a_{n-2} t_{i}^{n-3}+\cdots+2 a_{2} t_{i}+a_{1}$ (cf. eq. (5)).

The only difference is that now $N>n$ points are used for fitting the $n-1$ th order polynomial piece-wise to the time series. Since in this case we have $N-n$ equations, more than the number of unknowns, the system of equations can be solved by least squares adjustment.

In this study we fit a 7th order polynomial to 12 adjacent points and the derivative is determined at the middle point, then the whole procedure is moved one epoch further (moving window) for determining velocity at the next epoch. 


\subsection{Smoothing by Cubic Splines}

Splines were originally used for interpolation, described in section 3.3. They can be applied to smoothing by leaving a certain interval for the ordinate (position) of the spline at every epoch. Therefore in general the minimality criterion defined for spline interpolation is extended by a smoothing criterion.

Similarly to the interpolation, we consider a group of piecewise cubic functions, which are continuous; the continuity holds on the first and the second derivatives as well, i.e. $g\left(t_{i}\right)_{-}=$ $g\left(t_{i}\right)_{+}, \dot{g}\left(t_{i}\right)_{-}=\dot{g}\left(t_{i}\right)_{+}$and $\ddot{g}\left(t_{i}\right)_{-}=\ddot{g}\left(t_{i}\right)_{+}$at every $i$, and the third derivative is the step function, so $\ddot{g}\left(t_{i}\right)_{-} \neq \ddot{g}\left(t_{i}\right)_{+}$.

Among these functions we define the smoothing cubic spline by extending the minimality criterion of the spline interpolation with an additional one for smoothing. So, the smoothing cubic spline should minimize the second derivative

$$
\int_{t_{0}}^{t_{n}} \ddot{g}(t) \mathrm{d} t=\min
$$

such that

$$
\sum_{i=0}^{n}\left(\frac{g\left(t_{i}\right)-x_{i}}{\delta x_{i}}\right)^{2} \leq S
$$

The smoothing criterion is defined as a function of the variance of the offset between the real- and the smoothed ordinates, $g\left(t_{i}\right)-x_{i}$, weighted by the standard deviation, $\delta x_{i}$, and kept under a chosen smoothing parameter, $S$.

Finding the one and only solution of $g(t)$, the derivative of the function can be obtained as

$$
\dot{g}(t)=3 a_{i} t^{2}+2 b_{i} t+c_{i} .
$$

The exact formulations for the determination of the coefficients can be found in [6]. Further discussions on smoothing splines are given in [5].

\section{Numerical Tests}

For tests we have chosen one single day of the CHAMP kinematic orbit, day 200 (equivalent to 19th, July) of 2002 [7]. The day has been chosen by chance, and the data of this day by visual screening was found to be 'typical' among 'good' data, which means consistent data during the day with small data gaps only.

\subsection{Accuracy Test on Positions}

The first test is application of the numerical methods without taking the derivative, in order to test the numerical limitations of the techniques. So comparison of the approximated kinematic positions and the real position has been performed. As for the interpolation techniques, no relevant differences between them is expected; differences show numerical errors of these methods. For the smoothing methods the differences of the signals depend on the arbitrary chosen smoothing parameter, which is in case of the cubic spline smoothing defined directly, while in case of the polynomial fitting it is explicitly governed by the choice of the number of points and the order of the polynomial. The optimal
Tab. 1. RMS of kinematic position residuals obtained by different mathematical methods.

\begin{tabular}{ll}
\hline RMS & position residual [m] \\
\hline Numerical derivation & 1.9463 \\
Spline interpolation & 0.0 \\
Polynomial fitting (interpolation) & $1.3018 \times 10^{-6}$ \\
Newton-Gregory interpolation & $1.4228 \times 10^{-7}$ \\
Smoothing Splines & 2.1276 \\
Polynomial smoothing & 4.8747 \\
\hline
\end{tabular}

Tab. 2. Optimal scale factor for the smoothing parameter of the smoothing splines. The term 'position residuals' refer to kinematic minus smoothed positions, while 'velocity residuals' refers to reduced-dynamic minus smoothed velocities.

\begin{tabular}{ccc}
\hline $\begin{array}{c}\text { Scale } \\
\text { factor }\end{array}$ & $\begin{array}{c}\text { RMS position } \\
\text { residual [m] }\end{array}$ & $\begin{array}{c}\text { RMS velocity } \\
\text { residual [mm/s] }\end{array}$ \\
\hline 10 & 0.8667 & 0.3336 \\
40 & 1.7360 & 0.3334 \\
60 & 2.1276 & 0.3334 \\
100 & 2.7497 & 0.3335 \\
150 & 3.3714 & 0.3335 \\
200 & 3.8965 & 0.3337 \\
\hline
\end{tabular}

smoothing parameter for the smoothing spline is discussed later in this section.

Table 1 shows the RMS of the differences of analytically derived and real kinematic positions. The small RMS values of the interpolation techniques show that the methods are correctly implemented. The results for smoothing methods show that the room left for smoothing is in the range of some meters.

\subsection{The Optimal Smoothing Parameter for a Smoothing by Cubic Splines}

The smoothing extent is known to depend on the length of the time series [5], and also on the amplitude of the signal (sec. 3.6. The smoothing parameter was defined as a linear function of the length of the arcs, $S=$ scale_factor $\times$ length $(\operatorname{arc})$. We applied several smoothing spline functions to kinematic positions with different smoothing parameters, and the zeroth derivative (position) and determined the first derivative (velocity) of the smoothed function. Table 2 shows the RMS of the smoothed positions/velocities as function of the scale-factor, compared to the real kinematic positions/reduced-dynamic velocities, respectively. (In case of the velocity we have no value to compare the results to). A loose assumption: we assume that under-smoothed velocities would provide high RMS of the velocity residuals since all the noises are included, while over-smoothed velocities would again mean high RMS of the residuals, since parts of the signal is smoothed away. So the smoothed velocities are assumed to be the most realistic if they are the most similar to the reduced-dynamic velocities. This way we found the scale factor of 60 being the most accurate for the test day.

Keep in mind that the smoothing parameter is a function of the amplitude of the signal as well. Later on (see the next section) 
Tab. 3. RMS of kinematic velocity residuals obtained by different mathematical techniques. No reference orbit.

\begin{tabular}{lc}
\hline RMS & velocity residual $[\mathrm{mm} / \mathrm{s}]$ \\
\hline Numerical derivative & $129.765[\mathrm{~m} / \mathrm{s}]$ \\
Spline interpolation & 0.3334 \\
Polynomial fitting (interpolation) & 0.3350 \\
Newton-Gregory interpolation & 0.3102 \\
Smoothing Splines & 0.3334 \\
Polynomial smoothing & 0.2219 \\
\hline
\end{tabular}

when the smoothing is applied to position residuals (a much smaller signal), the smoothing parameter is defined as tenth of the length of the arc. Defining that smoothing parameter is more uncertain, since there is no possibility to define a reference for optimisation. In that case the reduced-dynamic velocities cannot be used for minimising the velocity residuals, since in the remove-restore step of kinematic velocity determination the reduced-dynamic orbit is used as the reference orbit (cf. section 4.3. Thus the smoothing parameter is defined empirically, by screening the smoothed and the real kinematic position residuals.

In Table 2 the RMS of the position residuals are increasing with increasing scale factors. This feature suggests that by smoothing not the high-frequency noise but the low-frequency signal is minimised. Fig. 2 a shows a sequence of the unsmoothed and the smoothed position residuals. Unfortunately the high-frequency information is unaltered, but the whole signal moves away from the reduced-dynamic positions (since the position residuals goes away from zero by increasing smoothing parameter). Fig. 2p shows the unsmoothed and the smoothed position residuals when the smoothing is done on position residuals. The figure shows that in this case the different smoothing parameters control different wavelengths of the smoothing, as to be expected. This figure shows the non-spectral-filter characteristics of the smoothing spline function: in case of a signal with a characteristically large amplitude at a certain frequency (i.e. the orbital frequency), the smoothing spline minimises the curvature at that frequency. However, in case of a nearly white spectral distribution of the data the smoothing starts to minimise at the highest frequencies.

\subsection{Velocity from the Full Position Signal}

Different interpolation and smoothing techniques for taking analytically the derivative of the position are first applied to purely kinematic positions. Fig. 3 shows the velocity residuals, i.e. the differences of the reduced-dynamic and the kinematic velocities. The following table (Table 3) shows the RMS of the velocity residuals.

Interpolation techniques provide all comparable accuracy for velocities. Newton-Gregory interpolation out-performed slightly the other techniques. As for the smoothing techniques, certain improvement was expected, which was actually delivered by the smoothing polynomial $(0.2219 \mathrm{~mm} / \mathrm{s})$. A range of
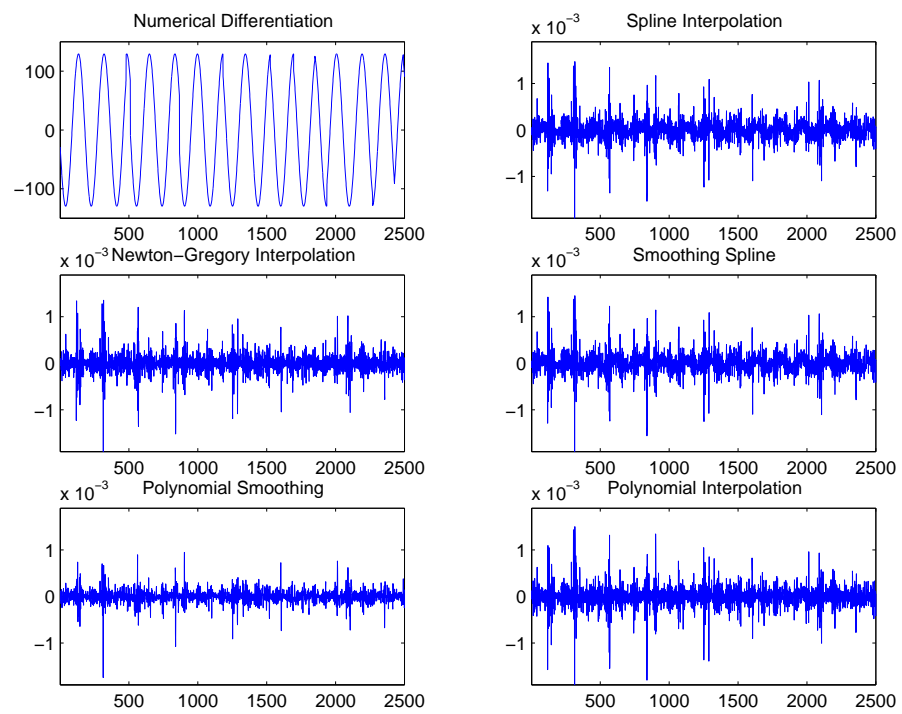

Fig. 3. Kinematic velocity residuals (i.e. minus reduced-dynamic velocity). For this solution no reference orbit was applied. (Abscissa: epochs [30 s], ordinate: velocity $[\mathrm{mm} / \mathrm{s}])$.

degrees of smoothing was tested for the smoothing spline, however those failed to improve the accuracy of the velocities compared to the spline interpolation (cf. also section 4.1).

\subsection{Velocity from Residual Position Signal}

With the hope of improving the accuracy, we have tried to reduce the amplitude of the signal by making use of a reference orbit in a remove-restore way. After removing the reference orbit, we took the derivatives on residuals (having an amplitude of some $\mathrm{cm}$ - instead of some thousands of $\mathrm{km}$ signal of the original position in the Earth-fixed system), finally adding back the residual kinematic velocity to the velocity of the reference orbit.

For reference orbits purely dynamic orbits have been determined, making use of the EIGEN-1S and the TEG-4 models, both up to degree and order of 120 . The reduced-dynamic CHAMP orbit was also used as a reference orbit. The reduceddynamic CHAMP orbit was based on the EIGEN-2 gravity model. The dynamic and reduced-dynamic positions were compared to the kinematic positions; the RMS of the position residuals was $1.1233 \mathrm{~m}, 1.1091 \mathrm{~m}$ and $0.0311 \mathrm{~m}$ for the dynamic EIGEN-1S, dynamic TEG-4 and reduced-dynamic EIGEN-2 orbits, respectively. Comparisons also has been done for the velocities: the EIGEN-1S and the TEG-4 velocities differ from the reduced-dynamic velocities with an RMS of $1.5826 \mathrm{~mm} / \mathrm{s}$ and $1.3562 \mathrm{~mm} / \mathrm{s}$, respectively.

Figs 4, 5b and 6 show the velocity residuals, the differences of the kinematic and the reduced-dynamic velocities. Tables $6-$ 6 show the RMS of the velocity residuals.

The results show no relevant dependence on the interpolation techniques - reflecting that the numerical errors of these techniques are less than $10^{-8}$-fold of the signal. It is noteworthy that the accuracy of velocities are slightly worse than in case of the no-reference-orbit velocity (cf. Table 3). This can be due to the less smooth characteristics of the signal. Although the posi- 
Fig. 2. Smoothed kinematic position residuals vs. real position residuals. Left frame: smoothing was done on the full position signal. Right frame: smoothing was done on position residuals.
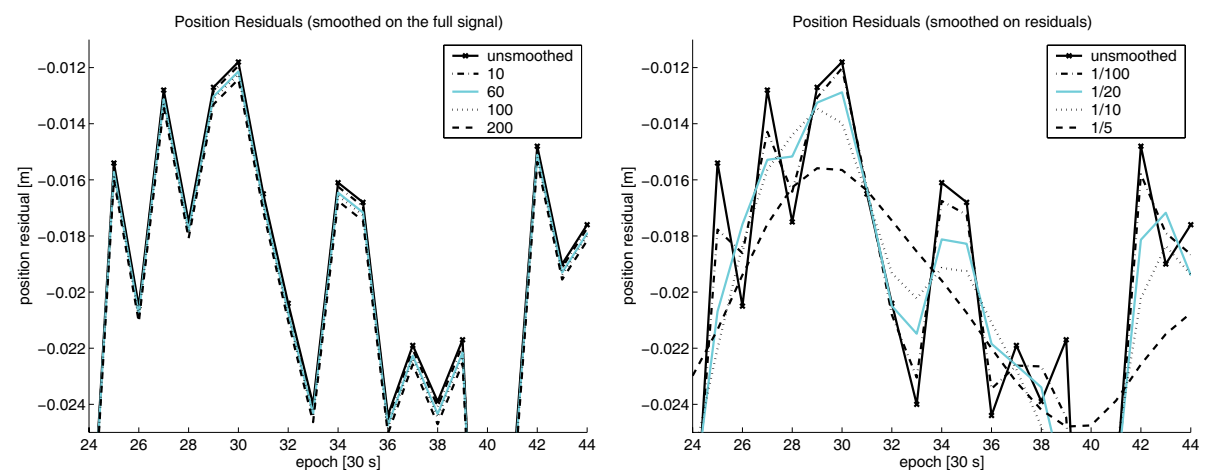
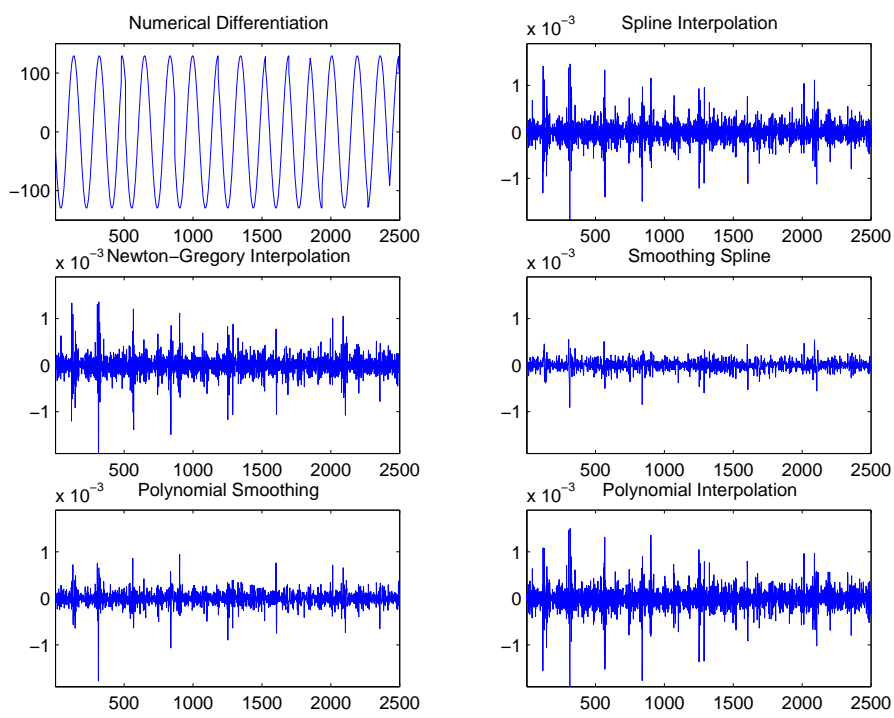

Fig. 4. Kinematic velocity residuals, applying an EIGEN-1S based dynamic orbit for reference. (Abscissa: epochs [30 s], ordinate: velocity [mm/s]).
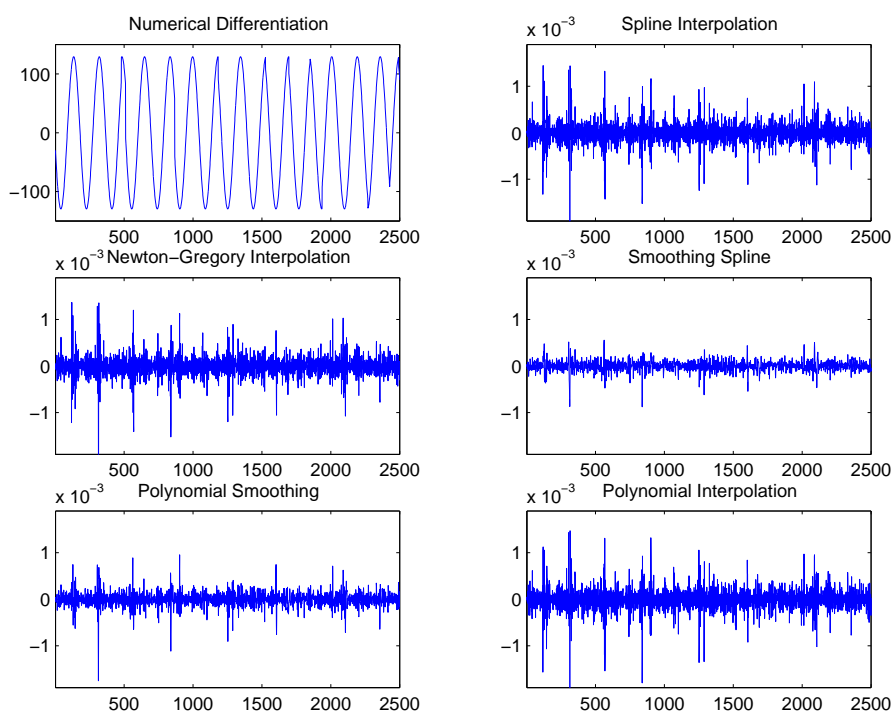

Fig. 5. Kinematic velocity residuals, applying a TEG-4 based dynamic orbit for reference. (Abscissa: epochs [30 s], ordinate: velocity [mm/s]).

tion residuals are drastically reduced, there is a large change in the curvatures of the function.

Smoothing techniques: similarly to interpolation, there is no significant change for the polynomial, however the smoothing spline improves a lot. For the polynomial, one can get lower RMS by altering the length of the arc for velocity estimation or
Tab. 6. RMS of kinematic velocity residuals obtained by different mathematical approximation techniques. Reference orbit: reduced-dynamic, EIGEN-2

\begin{tabular}{lc}
\hline RMS & velocity residual [mm/s] \\
\hline Numerical derivation & $129.765[\mathrm{~m} / \mathrm{s}]$ \\
Spline interpolation & 0.3356 \\
Polynomial fitting (interpolation) & 0.3465 \\
Newton-Gregory interpolation & 0.3185 \\
Smoothing Splines & 0.1554 \\
Polynomial smoothing & 0.2253 \\
\hline & \\
\hline RMS & velocity residual [mm/s] \\
\hline Numerical derivation & $129.765[\mathrm{~m} / \mathrm{s}]$ \\
Spline interpolation & 0.3384 \\
Polynomial fitting (interpolation) & 0.3492 \\
Newton-Gregory interpolation & 0.3213 \\
Smoothing Splines & 0.1541 \\
Polynomial smoothing & 0.2269 \\
\hline & \\
\hline RMS & 0.3505 \\
\hline Numerical derivation & 0.3220 \\
Spline interpolation & 0.1456 \\
Nolynomial fitting (interpolation) & 0.2255 \\
\hline Polynooth-Gregory interpolation & $0.767[\mathrm{~m} / \mathrm{s}]$ \\
\hline & \\
\hline
\end{tabular}

the order of the polynomial according to the less smoothed signal and its much smaller magnitude. A possible explanation of the large improvement using the smoothing splines in a removerestore sense is the nearly white spectral distribution of the signal. This should be analysed in the frequency domain (cf. [3]). Another possible explanation is that during the remove-restore step, the reference orbit affects the solution. Let us consider an obvious example for a false smoothing in a remove-restore step, where the output is dominated by the reference orbit. If one over-smoothes the position residuals, all the velocity residuals are smoothed to zero. Then the restore step adds zero to the reference orbit, providing the reference velocities without any change. We should prove therefore, (1) how our results differ from the reference, and (2) whether similar velocities result when different reference orbits are used. 

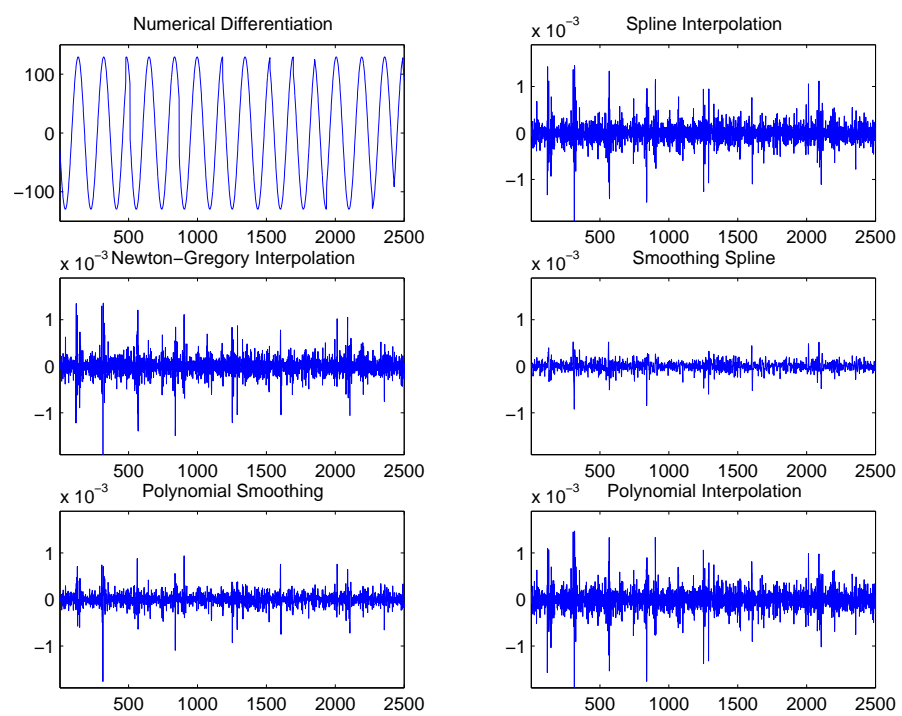

Fig. 6. Kinematic velocity residuals, applying the EIGEN-2 based reduceddynamic orbit for reference. (Abscissa: epochs [30 s], ordinate: velocity $[\mathrm{mm} / \mathrm{s}])$.

\section{Discussion}

For investigating whether our kinematic velocities are independent of the chosen reference orbit, the differently derived velocities and the different reference velocities are compared with each other. Table 7 contains RMS differences between velocities of different origin. These are:

1 'EIGEN1 dyn.': dynamic velocity, with EIGEN-1S model used for precise orbit determination (POD).

2 'TEG dyn': dynamic velocity, with TEG-4 model used for POD.

3 'EIGEN2 red-dyn.' : reduced-dynamic velocity, with EIGEN2 model used for POD. (Note: the reduced-dynamic orbit follows very closely the kinematic orbits, therefore the residuals are much smaller then in case of a dynamic orbit.)

4 'EIGEN1 kin.': kinematic velocity, using 'EIGEN1 dyn.' as reference for the velocity determination (cf. Table 6).

5 'TEG kin.': kinematic velocity, using 'TEG dyn' as reference for the velocity determination (cf. Table 6).

6 'EIGEN2 kin.': kinematic velocity, using 'EIGEN2 red-dyn.' as reference for the velocity determination (cf. Table 6).

7 'no-ref. kin.': kinematic velocity, derived from the full position signal, no reference orbit applied (cf. Table 3 ).

We compare first the RMS differences of table 7 with each other. They suggest no dependency of the kinematic velocities on the reference orbit. (1) Differences of the different dynamic velocities with each other and with the kinematic velocities (first 2 lines of the table) are the largest, showing some $1.0-1.6 \mathrm{~mm} / \mathrm{s}$ RMS between them. Excluding the last column (the no-reference orbit case) from the analysis one can recognise
Tab. 7. The RMS velocity differences between the different (reduced)dynamic and kinematic velocities $[\mathrm{mm} / \mathrm{s}]$.

\begin{tabular}{|c|c|c|c|c|c|c|}
\hline $\mathrm{RMS}$ [mm/s] & $\begin{array}{l}\text { TEG } \\
\text { dyn. }\end{array}$ & $\begin{array}{l}\text { EIGEN2 } \\
\text { red-dyn. }\end{array}$ & $\begin{array}{c}\text { EIGEN1 } \\
\text { kin. }\end{array}$ & $\begin{array}{l}\text { TEG } \\
\text { kin. }\end{array}$ & $\begin{array}{c}\text { EIGEN2 } \\
\text { kin. }\end{array}$ & $\begin{array}{c}\text { no-ref. } \\
\text { kin. }\end{array}$ \\
\hline EIGEN1 dyn. & 0.9661 & 1.5189 & 1.5622 & 1.5268 & 1.5259 & 1.5622 \\
\hline TEG dyn. & & 1.3036 & 1.3125 & 1.3104 & 1.3115 & 1.3564 \\
\hline EIGEN2 rd. & & & 0.1478 & 0.1458 & 0.1357 & 0.3876 \\
\hline EIGEN1 kin. & & & & 0.0615 & 0.0513 & 0.3399 \\
\hline TEG kin. & & & & & 0.0475 & 0.3386 \\
\hline EIGEN2 kin & & & & & & 0.3396 \\
\hline
\end{tabular}

the following: (2) The EIGEN2 reduced-dynamic RMS velocities are an order of magnitude smaller than the dynamic velocities (3rd line of the table, typically some $0.15 \mathrm{~mm} / \mathrm{s}$ ), (3) the CHAMP kinematic velocities of different origin show a similarity in size with each other $(0.04-0.06 \mathrm{~mm} / \mathrm{s}$; cf. $0.0615,0.0513$ and 0.0475 in the table). Thus we conclude that the kinematic velocities with different reference orbits used for velocity determination (EIGEN1 kin., TEG kin.) provide similar RMS values. Their size is smaller than any other kind of velocities (dynamic, reduced-dynamic), cf. the 5th and the 6th columns. The no-reference orbit solution is a different story. It shows a $0.35 \mathrm{~mm} / \mathrm{s}$ RMS similarity to the other kinematic sets, which is slightly smaller than RMS using the EIGEN2 reduced-dynamic orbit.

What is the absolute accuracy of a CHAMP velocity set? This question has been discussed at a workshop, the CHAMP Orbit Campaign in Potsdam held in 2002. The CHAMP reduceddynamic velocities of different institutes were found to differ only about $0.1 \mathrm{~mm} / \mathrm{s}$. These sets of reduced-dynamic velocities were derived by essentially independent techniques, so that we can assume that $0.1 \mathrm{~mm} / \mathrm{s}$ is a representative estimate of the accuracy of these reduced-dynamic velocities. According to this estimate, an accurate estimation of the kinematic velocities in absolute sense should not differ from the reduced-dynamic velocity (EIGEN2 red-dyn. in Table 7) by more than this threshold. In Table 7 all estimates for velocity exceed the $0.1 \mathrm{~mm} / \mathrm{s}$ threshold, particularly the no-reference orbit case seems to be unreliable $(0.3876 \mathrm{~mm} / \mathrm{s})$. At this point we should discuss the characteristics of these errors and their effect on the gravity analysis process. Probably the kinematic orbit contains systematic as well as random errors. The kinematic velocities are affected by errors of the kinematic positions. The different velocity estimation methods make the different epochs interdependent, because neighbouring points are involved in the velocity estimation. In the next processing step the velocity errors are squared in the energy integral when the disturbing potential is derived. The spherical harmonic analysis is done by Least Squares Adjustment. The observation equations are an expansion of the disturbing potential in terms of spherical harmonics. The disturbing potential is the vector of observables, $l$. It is multiplied by the inverse normal matrix, $x=N^{-1} l$, solving for the vector of unknowns, $x$, the spherical harmonic coefficients. This 
multiplication with the inverse normal matrix corresponds to a weighted summation of the observables over time. Random errors of the disturbing potential largely cancel while systematic errors are accumulated. Thus primarily the systematic errors of the disturbing potential have notable effects on the solution. By squaring the kinematic velocity errors for the computation of the disturbing potential, no correlation between different epochs has been taken into account. If the kinematic velocity errors are random, then the disturbing potential is random too. However, it is hard to assume that kinematic velocities are affected only by random errors, since we include always several (minimum two) neighbouring points for numerical derivation. So the numerical derivation introduces systematic errors. Also probably the kinematic positions are affected by systematic errors as well from the POD.

However there is no estimate available for the contribution of the systematic and of the random part of the errors. The systematic errors of POD and of the numerical derivation may be small compared to the signal, so velocities with a relatively high RMS difference from the reduced-dynamic velocities do not necessarily imply a bad solution for the spherical harmonic analysis. In the case of the no-reference orbit velocity one surely knows that (1) it is contaminated by the full error spectrum of the kinematic positions and (2) it is independent of any a priori gravity field.

The second property is of particular importance. The first one is interesting from a point of view of a probable weakness of the remove-restore technique. That is, smoothing techniques normally alter a certain bandwidth of the frequency spectrum. This means that (1) at some frequencies, e.g. once and twice per revolution, the velocity signal is dominated by the reference velocity, while at others it is undisturbed, and due to this (2) we introduce a systematic error by the smoothing. The first point should be analysed by investigations in the frequency domain [3], while the effect of the systematic errors can not really be investigated. What can be done, however, is to perform the full spherical harmonic analysis, and study the coefficients, whether they show relevant correlation with the input gravity field(s).

\section{Conclusion}

Finally we have tested two different techniques for kinematic velocity estimation. The difference is the way how the noise of the kinematic orbit is treated. This was the concept of deriving two gravity models at the Technical University of Munich (TUM) from CHAMP data.

We attempted to smooth out the noise by applying a smoothing by cubic spline functions 3.6 on position residuals. In this case we disturb the spectral characteristics of the orbit errors by introducing a systematic error by the fitting of the spline function. In this case the reference orbit (reduced-dynamic EIGEN2 orbit) also affects the solution - the extent of its effect is not known. Therefore further investigations should be done in the frequency domain, and it has been discussed in a separate paper
[3]. Velocities of this kind were used for the TUM-1S gravity model [4].

We also derived kinematic velocities by fitting a 7th order polynomial to the kinematic positions with the Newton-Gregory interpolation technique (3.4). In this case all noise is included, however, at least its spectral characteristic is unchanged. This solution should work out well, if the kinematic orbit errors are dominated by random errors, and systematic errors of the interpolation are less relevant. These velocities were used for the TUM-2Sp solution [2].

According to these two models, in the case of the CHAMP the latter solution was more successful [2]. However, it is obvious that this is case sensitive: for other satellites with less smooth orbit a smoothing can effectively provide successful filtering of the data.

\section{References}

1 Balmino G, Perosanz F, Rummel R, Sneeuw N, Sünkel H, CHAMP, GRACE and GOCE: mission concepts and simulations, Bolletino di Geofisica Teorica ed Applicata 40 (1999), 309-319.

2 Földváry L, Gerlach Ch, Švehla D, Frommknecht B, Gruber Th, Peters Th, Rothacher M, Rummel R, Sneeuw N, Steigenberger P, Gravity model TUM-2sp based on the energy balance approach and kinematic CHAMP orbits, Earth Observation with CHAMP - Results from Three Years in Orbit (Reigber Ch, Lühr H, Schwintzer P, Wickert J, eds.), Springer, Berlin, 2004, pp. 13-18.

3 Földváry L, Spectral Analysis of CHAMP Kinematic Velocities of LEOs Determined by Applying Smoothing Cubic Splines, Periodica Polytechnica Civil Engineering (2007), to appear.

4 Gerlach Ch, Földváry L, Švehla D, Gruber Th, Wermuth M, Rothacher M, Rummel R, Sneeuw N, Frommknecht B, Peters Th, Steigenberger P, A CHAMP only Gravity Field Model From Kinematic Orbits Using the Energy Integral, Geoph. Res. Letters 30(20) (2003), 2037, DOI 10.1029/2003GL018025.

5 Greville TNE (ed.), Thoery and Applications of Spline Functions: Proceedings of an Advanced Seminar Conducted by the Mathematic Research Center Academic Press, New York, London, 1969.

6 Reinsch Ch, Smoothing by Spline Functions, Numerische Mathematik 10 (1967), 177-183.

7 Švehla D, Rothacher M, CHAMP double-difference kinematic orbit with ambiguity resolution, First CHAMP Mission Results for Gravity, Magnetic and Atmospheric Studies (Reigber Ch, Lühr H, Schwintzer P, eds.), 2003, pp. 70-77. 